\section{Mortalidade e internações hospitalares por intoxicações e reações adversas a medicamentos no Brasil: análise de 2000 a 2014}

\author{
Hospitalizations and deaths from drug poisoning \\ and adverse reactions in Brazil: an analysis from \\ 2000 to 2014
}

\section{Mortalidad e internamientos hospitalarios por intoxicaciones y reacciones adversas a medicamentos en Brasil: análisis de 2000 a 2014}

Guidyan Anne Silva Santos 1

Alexandra Crispim Boing 1

\title{
Resumo
}

Nos últimos anos, as intoxicações e reações adversas a medicamentos tornaram-se importante causa de hospitalização e mortalidade, constituindo tema de grande relevância para a saúde pública. O objetivo deste estudo foi descrever a tendência de mortalidade e hospitalizações por esses agravos no Brasil, entre os anos de 2000 e 2014. Os dados utilizados foram provenientes dos sistemas SIM (Sistema de Informções sobre Mortalidade) e SIH-SUS (Sistema de Informações Hospitalares do SUS), e a população foi analisada segundo sexo, região de residência e faixa etária. Para definição dos eventos, foram utilizados códigos CID-10. A análise de tendência da série histórica foi realizada por regressão linear generalizada pelo método de Prais-Winsten, com correção do efeito de autocorrelação de primeira ordem. Tanto os óbitos quanto as hospitalizações ocorridas durante o período estudado apresentaram tendência ascendente. Na análise por regiões, as tendências mantiveram-se ascendentes para ambos os eventos nas regiões Sudeste e Sul. A análise por faixa etária mostrou tendências declinantes para menores de cinco anos nos dois eventos, tendência de óbitos ascendente para maiores de quatro anos e tendência de hospitalizações ascendente para a faixa de 20 a 59 anos. As intoxicações e reações adversas a medicamentos apresentam considerável contribuição para a ocorrência de óbitos e hospitalizações no Brasil, e os sistemas de informação SIM e SIH-SUS são fontes de dados de qualidade satisfatória para estudos de base populacional sobre mortalidade e morbidade hospitalar no país.

Intoxicação; Efeitos Colaterais e Reações Adversas Relacionados a Medicamento; Registros de Mortalidade; Sistemas de Informação Hospitalar

\author{
Correspondência \\ A. C. Boing \\ Universidade Federal de Santa Catarina. \\ Campus Universitário Reitor João David Ferreira Lima, \\ Florianopolis, SC 88040-970, Brasil. \\ acboing@gmail.com \\ 1 Universidade Federal de Santa Catarina, Florianópolis, Brasil.
}




\section{Introdução}

A importância dos medicamentos para a redução das taxas de mortalidade e morbidade das populações é um fato inegável na evolução da saúde atual, mas, apesar do crescimento dos recursos terapêuticos para o combate das enfermidades, há também o incremento no número de ocorrências relacionadas a eventos adversos a medicamentos, como as reações adversas a medicamentos e as intoxicações medicamentosas 1 .

Segundo a Organização Mundial da Saúde (OMS) 2, quaisquer ocorrências médicas desfavoráveis que possam ocorrer durante um tratamento medicamentoso são consideradas eventos adversos a medicamentos. Entre os eventos adversos a medicamentos, encontram-se as reações adversas a medicamentos e as intoxicações medicamentosas, ambas respostas indesejáveis decorrentes do uso de medicamentos, com a diferença de que, na primeira, as doses utilizadas são as usualmente empregadas, e, na segunda, as doses utilizadas são superiores às usuais. Assim, as reações adversas a medicamentos terão resposta normalmente não intencional, enquanto que as intoxicações medicamentosas poderão ter resposta acidental - relacionada a eventos de automedicação, erros de dosagem, terapêutica inadequada, confusão entre medicamentos e ingestão involuntária; ou intencional - relacionada a situações de abuso, uso indevido e autolesão 2,3.

Nas últimas décadas, diferentes estudos mostraram que a morbimortalidade relacionada a eventos medicamentosos adquiriu grande relevância como problema de saúde pública, tornando as questões de segurança do usuário importantes para o alcance de resultados terapêuticos eficazes e para a melhoria da qualidade de vida dos pacientes 4,5. A percepção do que é tido como seguro e as exigências de segurança dos medicamentos modificaram-se ao longo do tempo, tanto em função de avanços terapêuticos quanto de ocorrências desastrosas decorrentes do uso de medicamentos 6 . Isso fez com que incidentes com medicamentos tenham chamado a atenção de profissionais, instituições e autoridades sanitárias em todo o mundo, devido ao fato de contribuírem para o aumento da morbidade e da estada hospitalar, para a imposição de custos ao sistema de saúde e para alterações negativas na qualidade da assistência prestada 6,7 .

Nesse sentido, o número de estudos sobre a utilização de medicamentos e os problemas relacionados a essa prática têm crescido cada vez mais ao longo do tempo, juntamente com a frequência de ocorrências de eventos adversos a medicamentos. Em 2002, foi conduzida uma metanálise sobre internações hospitalares no mundo devido a eventos adversos a medicamentos e verificou-se que a prevalência de internações por esse motivo variou de $3 \%$ a $9 \%$, sendo que $50 \%$ dessas internações poderiam ter sido evitadas pelo uso racional dos medicamentos ${ }^{8}$. Uma análise de tendência das taxas de mortalidade por intoxicações medicamentosas entre os anos de 1994 e 2010, realizada nos Estados Unidos, relatou mais de 300 mil mortes, com taxas aumentando no decorrer do tempo de estudo em todas as regiões e grupos demográficos, e tendo como principal causa a intoxicação acidental, responsável por 71\% dos casos 9. O Annual Report of the American Association of Poison Control Centers 10 do ano de 2014 registrou mais de 2 milhões de exposições a agentes tóxicos, sendo que, entre as cinco principais classes de substâncias, três corresponderam a medicamentos: $11,3 \%$ das exposições estiveram relacionadas a analgésicos; 5,9\%, a sedativos/hipnóticos/antipsicóticos; e 4,4\%, a antidepressivos.

No Brasil, os resultados não são diferentes: os medicamentos estão entre as principais causas de intoxicação identificadas pelos Centros de Informação e Assistência Toxicológica (CIATs), ocupando a primeira posição entre os agentes causadores de intoxicação no país, à frente de tóxicos como raticidas e inseticidas, agrotóxicos, domissanitários, drogas ilícitas e alimentos impróprios para consumo 11. Dados registrados no Sistema Nacional de Informações Tóxico-Farmacológicas (SINITOX) apontam que, entre 2000 e 2012, foram notificados mais de 300 mil casos de intoxicações medicamentosas (aproximadamente 26.693 casos por ano), e, desses 1.102 (0,3\% dos casos) evoluíram para o óbito ${ }^{12}$. Paula et al. 13 observaram um crescimento de 13,6\% no número de hospitalizações em idosos por eventos adversos a medicamentos no país de 2004 para 2008, e Lieber \& Ribeiro 5 observaram que a prevalência de eventos adversos a medicamentos em crianças atendidas em serviços de emergência em São Paulo foi de 4\%, sendo que 0,1\% delas foram hospitalizadas por esse motivo. Entre 1996 e 2005, Mota et al. 14 observaram, no Brasil, uma taxa de mortalidade por intoxicações medicamentosas de 4,6 óbitos/10 mil registros, sendo 44\% dos óbitos ocorridos na faixa etária de 20 a 39 anos. 
Grande parte dos estudos nacionais sobre intoxicações medicamentosas e reações adversas a medicamentos tem abordado a caracterização dos perfis de mortalidade e internações hospitalares, relacionando variáveis como faixa etária e sexo do paciente, circunstância da intoxicação e classe terapêutica envolvida. Porém, a maioria dos estudos publicados utiliza, como fonte de dados, os registros disponíveis no SINITOX e nos bancos de dados dos CIATs, o que torna os estudos focados em espaços regionalizados. Além disso, alguns fatores colaboram para que essas informações tenham sua qualidade e quantidade comprometidas. A insuficiência do número de CIATs para cobrir toda a extensão territorial do país prejudica a regularidade da alimentação desses dados no SINITOX. Ainda o fato de a notificação ser espontânea gera falta de padronização entre os dados de diferentes CIATs e o desconhecimento por esse sistema das ocorrências atendidas diretamente na rede de serviço de saúde 14. Diante disso, ganha importância a realização de estudos que utilizem, como fonte de dados, os sistemas de informação em saúde disponíveis como o Sistema de Informações sobre Mortalidade (SIM) e o Sistema de Informações Hospitalares do Sistema Único de Saúde (SIH-SUS), ambos de abrangência nacional, notificação compulsória e qualidade satisfatória.

Nesse contexto, o objetivo do presente estudo foi descrever e analisar a tendência de mortalidade e internações hospitalares por intoxicações medicamentosas e reações adversas a medicamentos no Brasil, segundo sexo, região de residência e faixa etária, no período compreendido entre 2000 e 2014.

\section{Métodos}

Trata-se de um estudo descritivo, do tipo ecológico de série temporal, sobre as taxas de mortalidade e internações hospitalares causadas por intoxicações e reações adversas a medicamentos no Brasil durante o período de 2000 a 2014, segundo sexo, região de residência e faixa etária. Foram observadas também as informações sobre os códigos da 10a revisão da Classificação Internacional de Doenças (CID-10) envolvidos com os eventos estudados, segundo as variáveis descritivas utilizadas na análise.

Os dados sobre mortalidade e internações hospitalares utilizados foram provenientes dos sistemas de informação em saúde SIM e SIH-SUS. Os dados sobre o número de habitantes foram oriundos dos Censos de 2000 e 2010, das projeções da população de 2013 e 2014, e das estimativas intercensitárias para os demais anos, disponibilizadas pelo Instituto Brasileiro de Geografia e Estatística (IBGE. http://www.ibge.gov.br).

Os óbitos e internações hospitalares considerados para o estudo foram aqueles que apresentaram como causa de morte/condição adjacente ao óbito ou diagnóstico primário de internação hospitalar os seguintes códigos CID-10: relacionados a reações adversas a medicamentos - T88.6, T88.7 e Y40 aY57; e relacionados à intoxicação medicamentosa - F11.0, F13.0, F15.0, F19.0, F55, T36 a T39, T40.2 a T40.4, T41 a T50, X40, X41, X43, X44, X60, X61, X63, X64, X85, Y10, Y11, Y13 e Y14. Os códigos $\mathrm{X} 42$, $\mathrm{X} 62$ e $\mathrm{Y} 12$ foram suprimidos, para evitar a seleção de códigos que não permitam diferenciar as intoxicações causadas por medicamentos daquelas causadas por drogas lícitas e ilícitas, conforme utilizado por Paula et al. 13 e Lessa \& Bochner 15. As circunstâncias de exposição para as intoxicações medicamentosas estudadas foram obtidas pelos códigos X40 a X44 (circunstância acidental), X60 a X64 (autointoxicação), X85 (agressão) e Y10 a Y14 (circunstância não determinada).

A análise dos óbitos e hospitalizações estudados foi realizada segundo o sexo (feminino e masculino), a região de residência (Norte, Nordeste, Centro-oeste, Sudeste e Sul do Brasil) e a faixa etária (0 a 4 anos, 5 a 19 anos, 20 a 59 anos e 60 anos ou mais). As taxas brutas de mortalidade/internação hospitalar foram calculadas dividindo-se o número de óbitos/internações hospitalares ocorridos na população de estudo pelo número de habitantes correspondente ao período. Como as taxas brutas são muito influenciadas pela estrutura etária e distribuição por sexo das populações de diferentes regiões e entre diferentes anos, as taxas calculadas foram padronizadas pelo método direto por sexo e faixa etária, utilizando-se, como padrão, a população mundial sugerida pela OMS para que não haja interferência na estimação de tendências e na comparação de dados 16 .

A análise de tendência da série histórica foi realizada por regressão linear generalizada pelo método de Prais-Winsten, com correção para o efeito de autocorrelação de primeira ordem, utilizando-se o software Stata 13 (StataCorp LP, College Station, Estados Unidos). A tendência de óbitos/internações hospitalares foi considerada estacionária quando $\mathrm{p}>0,05$; declinante quando $\mathrm{p}<0,05$ e coeficiente 
da regressão negativo; ou ascendente quando $\mathrm{p}<0,05$ e coeficiente da regressão positivo. Para evitar rugosidades no polígono delineado nos gráficos, causadas pela variação aleatória na série temporal, foi utilizado o recurso de alisamento por médias móveis de ordem 317.

\section{Resultados}

Entre os anos de 2000 e 2014, aproximadamente, 0,1\% dos óbitos e 0,4\% das internações hospitalares ocorridas no Brasil tiveram como causa intoxicações e reações adversas a medicamentos. Em sua maioria, esses eventos estiveram relacionados a intoxicações medicamentosas ( $88 \%$ dos óbitos e 99,5\% das hospitalizações), superando as ocorrências relacionadas a reações adversas a medicamentos. A taxa de mortalidade por esses eventos praticamente dobrou durante o período estudado, passando de 2,8 óbitos/1 milhão de habitantes no ano de 2000 para 5,5 óbitos/1 milhão de habitantes em 2014. A taxa de hospitalizações aumentou em 1,6 vez, passando de 14,4 hospitalizações/100 mil habitantes em 2000 para 23,8 hospitalizações/100 mil habitantes em 2014.

As taxas médias de óbitos e internações hospitalares apresentaram-se maiores no sexo masculino durante o período estudado, tanto no Brasil (4,40 vs. 3,59 óbitos/1 milhão de habitantes e 34,43 vs. 12,90 hospitalizações/100 mil habitantes) quanto em suas regiões (em média, as taxas de óbito foram 1,3 vez maiores no sexo masculino, e as taxas de hospitalização, 2,6 vezes maiores). Somente a taxa média de óbito na Região Centro-oeste apresentou-se maior no sexo feminino (Tabela 1). As maiores taxas médias de óbito e internação hospitalar ocorreram nas regiões Centro-oeste e Sul, respectivamente (5,27 óbitos/1 milhão de habitantes e 41,10 hospitalizações/100 mil habitantes), seguida das regiões Sul, Sudeste, Nordeste e Norte para óbitos; e Centro-oeste, Sudeste, Nordeste e Norte para internações hospitalares, como mostra a Tabela 1.

$\mathrm{Na}$ análise segundo a faixa etária, foram utilizadas as taxas médias brutas de óbitos e hospitalizações para o Brasil e suas regiões. Os resultados mostraram que, tanto no Brasil quanto em suas regiões, as menores taxas de mortalidade ocorreram principalmente na faixa etária de 0 a 4 anos, e de internações hospitalares, na faixa etária de 60 anos ou mais. As maiores taxas de mortalidade foram encontradas na faixa etária de 60 anos ou mais $(7,2$ vezes maior do que a faixa etária de 0 a 4 anos) e de internação hospitalar, na faixa etária de 20 a 59 anos (4,1 vezes maior do que a faixa etária de 60 anos ou mais).

A análise dos códigos CID-10 considerados mostra como principal causa de óbito a autointoxicação por exposição intencional a outras drogas, medicamentos e substâncias biológicas não especificadas (X64) (21,7\%). Para hospitalizações, a principal causa encontrada foi a intoxicação aguda pelo uso de múltiplas drogas e outras substâncias psicoativas (F19) (68,1\%). A análise das taxas observadas nas faixas etárias de maior expressão nos resultados mostra como principais causas de óbito na faixa etária de 60 anos ou mais a intoxicação por exposição de intenção indeterminada a outras drogas, medicamentos e substâncias biológicas não especificadas (Y14) (16\%); seguida pelos efeitos adversos de outras drogas e medicamentos não especificados (Y57) (12,3\%); e como terceira principal causa a autointoxicação por exposição intencional a outras drogas, medicamentos e substâncias biológicas não especificadas (X64) (11,1\%). Para as internações hospitalares na faixa etária de 20 a 59 anos, as principais causas incluem a intoxicação aguda pelo uso de múltiplas drogas e outras substâncias psicoativas (F19) (74,9\%); em segundo lugar, a intoxicação por diuréticos e outras drogas, medicamentos e substâncias biológicas (T50) (5,7\%); e, após essa, a intoxicação por antibióticos sistêmicos (T36) (3,8\%).

As principais causas de óbito pelos eventos estudados nas demais faixas etárias corresponderam a: intoxicação acidental por outras drogas, medicamentos e substâncias biológicas não especificadas (X44) em crianças de 0 a 4 anos (32,2\%); e autointoxicação por exposição intencional a outras drogas, medicamentos e substâncias biológicas não especificadas (X64) nas faixas etárias de 5 a 19 anos e 20 a 59 anos (28,8\% e $24 \%$, respectivamente). Para as internações hospitalares, as principais causas incluíram: intoxicação por diuréticos e outras drogas, medicamentos e substâncias biológicas (T50) em crianças de 0 a 4 anos (30,9\%) e em idosos de 60 anos ou mais (21\%); e intoxicação aguda pelo uso de múltiplas drogas e outras substâncias psicoativas (F19) na faixa etária de 5 a 19 anos (60,7\%).

$\mathrm{Na}$ análise das causas segundo o sexo, os homens morreram principalmente devido à intoxicação aguda pelo uso de múltiplas drogas e outras substâncias psicoativas (F19), e as mulheres, devido à 
Tabela 1

Taxas médias de mortalidade (por 1 milhão de habitantes) e hospitalizações (por 100 mil habitantes) por intoxicações e reações adversas a medicamentos, segundo sexo. Brasil e regiões, 2000-2014.

\begin{tabular}{|c|c|c|c|c|c|c|}
\hline & Brasil & Norte & Nordeste & Centro-oeste & Sudeste & Sul \\
\hline \multicolumn{7}{|l|}{ Óbitos } \\
\hline \multicolumn{7}{|l|}{ Sexo } \\
\hline Masculino & 4,40 & 3,10 & 4,04 & 5,08 & 4,32 & 5,54 \\
\hline Feminino & 3,59 & 2,09 & 2,63 & 5,49 & 3,73 & 4,68 \\
\hline Ambos & 3,98 & 2,60 & 3,31 & 5,27 & 4,01 & 5,10 \\
\hline \multicolumn{7}{|c|}{ Hospitalizações } \\
\hline \multicolumn{7}{|l|}{ Sexo } \\
\hline Masculino & 34,43 & 6,92 & 22,50 & 40,40 & 35,95 & 63,55 \\
\hline Feminino & 12,90 & 3,87 & 7,15 & 16,69 & 15,56 & 19,15 \\
\hline Ambos & 23,46 & 5,41 & 14,61 & 28,32 & 25,52 & 41,10 \\
\hline
\end{tabular}

Fontes: Instituto Brasileiro de Geografia e Estatística (IBGE), Sistema de Informações Hospitalares do SUS (SIH-SUS) e Sistema de Informações sobre Mortalidade (SIM).

autointoxicação por exposição intencional a anticonvulsivantes, sedativos, hipnóticos, antiparkinsonianos e psicotrópicos (X61) (19,5\% e 20,2\%, respectivamente). Nas internações hospitalares, ambos os sexos tiveram como principal causa a intoxicação aguda pelo uso de múltiplas drogas e outras substâncias psicoativas (F19) (79,6\% dos homens e 37,4\% das mulheres).

$\mathrm{Na}$ análise de tendência, tanto para óbitos quanto para internações hospitalares por intoxicações e reações adversas a medicamentos no Brasil, as tendências apresentaram-se ascendentes, em ambos os sexos. A análise por regiões mostra a Região Norte com tendência estacionária para óbitos e ascendente para hospitalizações; a Região Nordeste com tendência estacionária para ambos os eventos; a Região Centro-oeste com tendência ascendente para óbitos e estacionária para hospitalizações; e as regiões Sudeste e Sul com tendência ascendente para ambos os eventos (Figura 1). É possível observar, nas séries históricas das taxas de mortalidade e de hospitalizações no Brasil durante o período estudado, a predominância de ocorrências nas regiões do Centro-Sul, sempre muito próximas ou acima das ocorrências nacionais. As regiões Norte e Nordeste mantiveram-se abaixo das ocorrências nacionais para os dois eventos.

Foram observadas diferenças por sexo nas regiões Norte, Nordeste e Centro-oeste. As duas últimas apresentaram tendência de mortalidade ascendente para o sexo masculino e estacionária para o sexo feminino, enquanto que a primeira apresentou tendência de hospitalizações ascendente para o sexo masculino e estacionária para o sexo feminino (Tabela 2).

A análise de tendência por faixa etária apresentou tendência de óbito ascendente para maiores de quatro anos e declinante para menores de cinco anos durante o período estudado. Para hospitalizações, a tendência apresentou-se ascendente para a faixa etária de 20 a 59 anos e, assim como a tendência de óbitos, declinante para os menores de cinco anos (Tabela 3).

A análise de tendência realizada separadamente para intoxicações medicamentosas e reações adversas a medicamentos apresentou tendências de óbito e de hospitalização ascendentes para intoxicações medicamentosas durante o período estudado. Para reações adversas a medicamentos, as tendências apresentaram-se estacionárias para os dois eventos (Tabela 4).

\section{Discussão}

O estudo identificou aumento das taxas de óbito e hospitalização por intoxicações e reações adversas a medicamentos no Brasil durante o período estudado, as quais se apresentaram aproximadamente duas vezes maiores em 2014 quando comparadas ao ano de 2000, ano de início da análise. As proporções de óbitos e internações hospitalares encontradas $(0,1 \%$ e $0,4 \%$, respectivamente) foram próximas 
Figura 1

Série histórica das taxas de mortalidade (1a) e hospitalizações (1b) por reações adversas a medicamentos. Brasil e regiões, $2000-2014$.

1a) Mortalidade

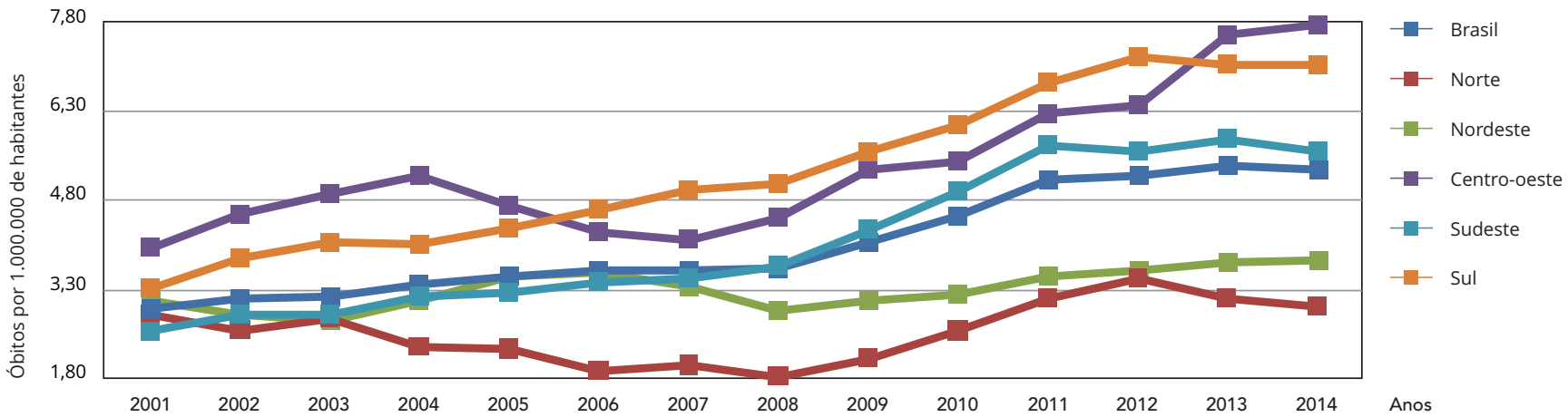

1b) Hospitalizações

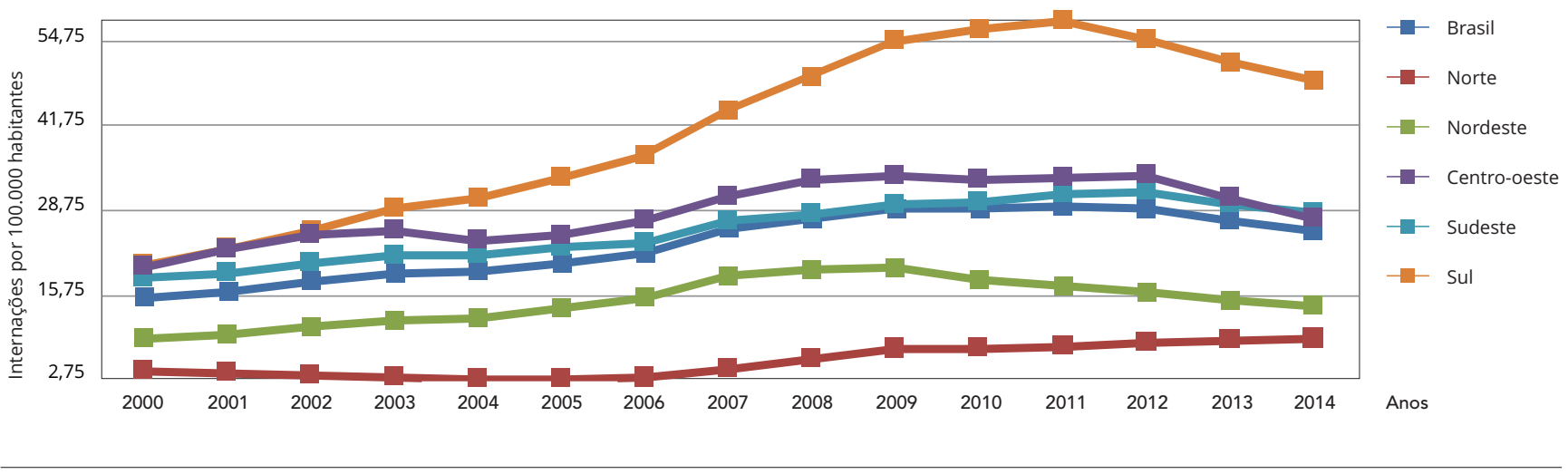

às descritas em estudos anteriores $8,14,18,19$ realizados no Brasil e no mundo sobre a ocorrência de eventos relacionados a intoxicações medicamentosas ou a reações adversas a medicamentos. Porém, devem ser consideradas as diferenças metodológicas entre os estudos, especialmente quanto à terminologia considerada para o desfecho e quantos às unidades territoriais observadas.

Mota et al. 14, em seu estudo sobre mortalidade por intoxicações medicamentosas no Brasil, encontraram uma proporção de óbitos de 0,04\% entre 1996 e 2005, sendo essa 2,5 vezes menor do que a proporção encontrada neste estudo. Essa diferença pode ser explicada pelo fato de não terem sido considerados por Mota et al. 14 os óbitos causados por reações adversas a medicamentos, e uma vez que a maioria dos casos considerados neste estudo trata-se de intoxicações medicamentosas, esse resultado pode caracterizar a ocorrência de óbitos por intoxicação medicamentosa ascendente ao longo do tempo no país. No âmbito internacional, metanálises 8,18,19 sobre hospitalizações por intoxicações medicamentosas ou reações adversas a medicamentos apresentaram prevalências superiores ou semelhantes às encontradas nesta pesquisa.

A metanálise realizada por Winterstein et al. 8 selecionou 15 estudos realizados na Alemanha, Austrália, Canadá, Dinamarca, Estados Unidos, França, Inglaterra e Itália, os quais apresentaram prevalências de internações por problemas relacionados a medicamentos variando entre 3\% e 9\%, valores esses superiores ao identificado no presente estudo. Isso pode ser explicado pela diferença na 
Tabela 2

Óbitos e hospitalizações, coeficiente de regressão e tendência de mortalidade e hospitalizações por intoxicações e reações adversas a medicamentos, segundo o sexo. Brasil e regiões, 2000-2014.

\begin{tabular}{|c|c|c|c|c|c|}
\hline \multicolumn{6}{|c|}{ Brasil } \\
\hline Sexo & Óbitos & Coeficiente & IC95\% & Valor de $p$ & Tendência \\
\hline Masculino & 5.907 & 0,25 & 0,$16 ; 0,35$ & $<0,001$ & Ascendente \\
\hline Feminino & 5.111 & 0,14 & 0,$10 ; 0,17$ & $<0,001$ & Ascendente \\
\hline Ambos & 11.018 & 0,20 & 0,$14 ; 0,25$ & $<0,001$ & Ascendente \\
\hline Sexo & Hospitalizações & Coeficiente & IC95\% & Valor de $p$ & Tendência \\
\hline Masculino & 485.830 & 0,62 & 0,$07 ; .2,76$ & 0,041 & Ascendente \\
\hline Feminino & 185.704 & 0,22 & 0,$02 ; 0,42$ & 0,032 & Ascendente \\
\hline Ambos & 671.534 & 0,81 & 0,$05 ; 1,56$ & 0,038 & Ascendente \\
\hline \multicolumn{6}{|c|}{ Região Norte } \\
\hline Sexo & Óbitos & Coeficiente & IC95\% & Valor de $p$ & Tendência \\
\hline Masculino & 313 & 0,05 & $-0,09 ; 0,19$ & 0,448 & Estacionária \\
\hline Feminino & 213 & $-0,01$ & $-0,10 ; 0,07$ & 0,827 & Estacionária \\
\hline Ambos & 526 & 0,01 & $-0,10 ; 0,12$ & 0,808 & Estacionária \\
\hline Sexo & Hospitalizações & Coeficiente & IC95\% & Valor de $p$ & Tendência \\
\hline Masculino & 7.861 & 0,63 & 0,$22 ; 1,04$ & 0,005 & Ascendente \\
\hline Feminino & 4.315 & 0,17 & $-0,00 ; 0,34$ & 0,055 & Estacionária \\
\hline Ambos & 12.167 & 0,39 & 0,$10 ; 0,68$ & 0,012 & Ascendente \\
\hline \multicolumn{6}{|c|}{ Região Nordeste } \\
\hline Sexo & Óbitos & Coeficiente & IC95\% & Valor de $p$ & Tendência \\
\hline Masculino & 1.421 & 0,10 & 0,$01 ; 0,18$ & 0,023 & Ascendente \\
\hline Feminino & 1.002 & 0,00 & $-0,05 ; 0,05$ & 0,972 & Estacionária \\
\hline Ambos & 2.423 & 0,04 & $-0,02 ; 0,11$ & 0,142 & Estacionária \\
\hline Sexo & Hospitalizações & Coeficiente & IC95\% & Valor de $p$ & Tendência \\
\hline Masculino & 84.355 & 0,78 & $-0,56 ; 2,09$ & 0,232 & Estacionária \\
\hline Feminino & 28.337 & 0,00 & $-0,27 ; 0,27$ & 0,994 & Estacionária \\
\hline Ambos & 112.692 & 0,37 & $-0,39 ; 1,14$ & 0,313 & Estacionária \\
\hline \multicolumn{6}{|c|}{ Região Centro-oeste } \\
\hline Sexo & Óbitos & Coeficiente & IC95\% & Valor de $p$ & Tendência \\
\hline Masculino & 502 & 0,37 & 0,$23 ; 0,50$ & $<0,001$ & Ascendente \\
\hline Feminino & 537 & 0,18 & $-0,01 ; 0,38$ & 0,057 & Estacionária \\
\hline Ambos & 1.039 & 0,27 & 0,$11 ; 0,43$ & 0,003 & Ascendente \\
\hline Sexo & Hospitalizações & Coeficiente & IC95\% & Valor de $p$ & Tendência \\
\hline Masculino & 42.076 & 1,37 & $-0,03 ; 3,07$ & 0,106 & Estacionária \\
\hline Feminino & 16.827 & $-0,05$ & $-0,26 ; 0,15$ & 0,577 & Estacionária \\
\hline Ambos & 58.903 & 0,64 & $-0,30 ; 1,58$ & 0,166 & Estacionária \\
\hline \multicolumn{6}{|c|}{ Região Sudeste } \\
\hline Sexo & Óbitos & Coeficiente & IC95\% & Valor de $p$ & Tendência \\
\hline Masculino & 2.540 & 0,34 & 0,$22 ; 0,46$ & $<0,001$ & Ascendente \\
\hline Feminino & 2.357 & 0,20 & 0,$15 ; 0,24$ & $<0,001$ & Ascendente \\
\hline Ambos & 4.897 & 0,27 & 0,$20 ; 0,35$ & $<0,001$ & Ascendente \\
\hline Sexo & Hospitalizações & Coeficiente & IC95\% & Valor de $p$ & Tendência \\
\hline Masculino & 217.821 & 1,46 & 0,$54 ; 2,38$ & 0,005 & Ascendente \\
\hline Feminino & 96.026 & 0,29 & 0,$19 ; 0,39$ & $<0,001$ & Ascendente \\
\hline Ambos & 313.847 & 0,89 & 0,$45 ; 1,33$ & 0,001 & Ascendente \\
\hline
\end{tabular}

(continua) 
Tabela 2 (continuação)

\begin{tabular}{|c|c|c|c|c|c|}
\hline \multicolumn{6}{|c|}{ Região Sul } \\
\hline Sexo & Óbitos & Coeficiente & IC95\% & Valor de $p$ & Tendência \\
\hline Masculino & 1.131 & 0,37 & 0,$23 ; 0,51$ & $<0,001$ & Ascendente \\
\hline Feminino & 1.002 & 0,27 & 0,$22 ; 0,32$ & $<0,001$ & Ascendente \\
\hline Ambos & 2.113 & 0,32 & 0,$26 ; 0,38$ & $<0,001$ & Ascendente \\
\hline Sexo & Hospitalizações & Coeficiente & IC95\% & Valor de $p$ & Tendência \\
\hline Masculino & 133.717 & 3,70 & 1,$00 ; 6,41$ & 0,011 & Ascendente \\
\hline Feminino & 40.199 & 0,70 & 0,$09 ; 1,30$ & 0,028 & Ascendente \\
\hline Ambos & 173.916 & 2,18 & 0,$55 ; 3,80$ & 0,013 & Ascendente \\
\hline
\end{tabular}

IC95\%: intervalo de 95\% de confinaça.

Fontes: Instituto Brasileiro de Geografia e Estatística (IBGE), Sistema de Informações Hospitalares do SUS (SIH-SUS) e Sistema de Informações sobre Mortalidade (SIM).

Tabela 3

Óbitos e hospitalizações, coeficiente de regressão e tendência de mortalidade e hospitalizações por intoxicações e reações adversas a medicamentos, segundo a faixa etária. Brasil, 2000-2014.

\begin{tabular}{lccccc}
\hline \multicolumn{5}{c}{ Brasil } \\
\hline Faixa etária (anos) & Óbitos & Coeficiente & $\mathbf{I C 9 5 \%}$ & Valor de $\mathbf{p}$ & Tendência \\
$0-4$ & 230 & $-0,03$ & $-0,06 ;-0,10$ & 0,012 & Declinante \\
$5-19$ & 889 & 0,03 & 0,$01 ; 0,06$ & 0,016 & Ascendente \\
$20-59$ & 8.012 & 0,32 & 0,$24 ; 0,40$ & $<0,001$ & Ascendente \\
60 ou mais & 1.887 & 0,18 & 0,$07 ; 0,28$ & 0,003 & Ascendente \\
Faixa etária (anos) & Hospitalizações & Coeficiente & $\mathbf{I C 9 5 \%}$ & Valor de $\mathbf{p}$ & Tendência \\
0-4 & 22.939 & $-0,26$ & $-0,44 ;-0,08$ & 0,007 & Declinante \\
5-19 & 104.059 & 0,19 & $-0,25 ; 0,64$ & 0,369 & Estacionária \\
$20-59$ & 522.355 & 1,48 & 0,$27 ; 2,69$ & 0,020 & Ascendente \\
60 ou mais & 22.181 & $-0,14$ & $-0,27 ; 0,00$ & 0,052 & Estacionária \\
\hline
\end{tabular}

IC95\%: intervalo de 95\% de confinaça.

Fontes: Instituto Brasileiro de Geografia e Estatística (IBGE), Sistema de Informações Hospitalares do SUS (SIH-SUS) e Sistema de Informações sobre Mortalidade (SIM).

definição do desfecho utilizado, uma vez que os problemas relacionados a medicamentos abrangem outras situações além de intoxicações medicamentosas e reações adversas a medicamentos. Porém, outra metanálise 18 sobre hospitalizações, dessa vez apenas por reações adversas a medicamentos, selecionou 68 estudos contemplando os países África do Sul, Austrália, Canadá, Croácia, Dinamarca, Espanha, Estados Unidos, França, China, Holanda, Inglaterra, Irã, Israel, Itália, Líbano, Suécia e Suíça, e identificou prevalências no mundo variando entre 0,2\% e 41,3\%; estando portanto a proporção encontrada neste estudo dentro dos limites observados. Outra metanálise mais recente sobre hospitalizações 19 também apenas por reações adversas a medicamentos na Europa - 47 estudos nos países Alemanha, Áustria, Eslovênia, Espanha, Finlândia, França, Grécia, Holanda, Inglaterra, Itália, Noruega, Romênia, Suécia e Suíça - apresentou prevalências variando entre 0,5\% e 12,8\%, com limite inferior discretamente maior do que a prevalência encontrada neste estudo. Nesses casos em que foram considerados apenas eventos envolvendo reações adversas a medicamentos, deve ser lembrado que essas compreenderam uma pequena parte das internações estudadas nesta análise (apenas 0,5\%). Assim, se fossem consideradas apenas as hospitalizações por reações adversas a medicamentos deste estudo, a prevalência encontrada estaria abaixo dos limites inferiores das duas últimas metanálises citadas 18,19 . 
Tabela 4

Óbitos e hospitalizações, coeficiente de regressão e tendência de mortalidade e hospitalizações por intoxicações e reações adversas a medicamentos, classificados em intoxicações medicamentosas e reações adversas a medicamentos. Brasil, 2000-2014.

\begin{tabular}{|c|c|c|c|c|c|}
\hline & \multicolumn{5}{|c|}{ Brasil } \\
\hline & Óbitos & Coeficiente & IC95\% & Valor de p & Tendência \\
\hline Intoxicações medicamentosas & 9.700 & 0,41 & 0,$31 ; 0,51$ & $<0,001$ & Ascendente \\
\hline Reações adversas a medicamentos & 1.318 & $-0,02$ & $-0,04 ; 0,01$ & 0,144 & Estacionária \\
\hline \multirow[t]{2}{*}{ Ambos } & 11.018 & 0,20 & 0,$14 ; 0,25$ & $<0,001$ & Ascendente \\
\hline & Hospitalizações & Coeficiente & IC95\% & Valor de $p$ & Tendência \\
\hline Intoxicações medicamentosas & 668.108 & 1,66 & 0,$19 ; 3,13$ & 0,029 & Ascendente \\
\hline Reações adversas a medicamentos & 3.426 & $-0,04$ & $-0,10 ; 0,02$ & 0,142 & Estacionária \\
\hline Ambos & 671.534 & 0,81 & 0,$05 ; 1,56$ & 0,038 & Ascendente \\
\hline
\end{tabular}

IC95\%: intervalo de 95\% de confinaça.

Fontes: Instituto Brasileiro de Geografia e Estatística (IBGE), Sistema de Informações Hospitalares do SUS (SIH-SUS) e Sistema de Informações sobre Mortalidade (SIM).

A predominância de óbitos e hospitalizações causados por intoxicações medicamentosas sobre os causados por reações adversas a medicamentos demonstra a característica evitável dos agravos no país, uma vez que as intoxicações medicamentosas ocorrem quando há sobredosagem, em circunstâncias acidentais ou intencionais. Além disso, deve ser considerado que, muitas vezes, são diagnosticadas apenas as reações adversas a medicamentos que causam grandes danos ao paciente, desconsiderando as de menor proporção 20,21. Isso pode gerar uma maior subnotificação de reações adversas a medicamentos quando comparadas às intoxicações medicamentosas, essas geralmente mais graves do que as primeiras. A característica evitável dos agravos fica evidente quando são observadas as principais causas de óbitos e hospitalizações encontradas - a maioria dos óbitos ocorreu por autointoxicação intencional e das internações por intoxicação aguda por múltiplas drogas ou substâncias psicoativas. Essa observação é condizente com a encontrada por Mota et al. 14 em que a principal circunstância de óbito por intoxicações medicamentosas encontrada no Brasil foi a tentativa de suicídio $(57,2 \%)$. Estudos sobre consumo de medicamentos 22,23,24,25,26,27,28 indicam que o perfil epidemiológico brasileiro atual é caracterizado pela alta prevalência de doenças crônicas, muitas vezes concomitantes, o que leva ao uso de múltiplos medicamentos. Essa pode ser uma das causas da ascensão de internações hospitalares por intoxicação por múltiplas substâncias no país.

Alguns autores afirmam que grande parte dos casos de reações adversas a medicamentos poderia ser evitada pela simples melhoria da qualidade das prescrições 29,30 ou da prevenção de problemas relacionados à adesão dos pacientes ao tratamento medicamentoso e prevenção da prática inapropriada de automedicação 31. Nesse contexto, a baixa prevalência de óbitos e hospitalizações por reações adversas a medicamentos encontrada neste estudo pode refletir uma melhoria no processo de prescrição, adesão ao tratamento e prevenção da automedicação inapropriada no Brasil, durante o período observado. Ainda é importante considerar a possibilidade da subnotificação dos eventos relacionados a reações adversas a medicamentos, conforme discutido no parágrafo anterior. Por outro lado, a elevada ocorrência de hospitalizações por intoxicação aguda por múltiplas substâncias pode indicar atenção insuficiente às interações medicamentosas. As interações podem ocorrer por descuido na administração dos medicamentos, porém, também podem ser decorrentes de falhas no momento da prescrição, uma vez que a ausência de anamnese adequada e observação prévia do histórico do paciente podem ocasionar prescrições equivocadas, permitindo a ocorrência de respostas indesejáveis.

$\mathrm{Na}$ análise por sexo, os homens lideraram as ocorrências tanto para hospitalizações quanto para óbitos. Esse dado pode ser conflitante quando confrontado com estudos sobre consumo de medicamentos realizados no Brasil, os quais mostram maiores prevalências entre indivíduos do sexo feminino 32,33,34. Porém, estudos apontam que aspectos socioculturais e biológicos favorecem o adoecimento 
de mulheres e que essas apresentam maior autocuidado e busca por serviços de saúde 35. É possível associar esse fato à maior ocorrência de hospitalizações observada em homens, pois a ausência de autocuidado e de busca por serviços de saúde pode levar a práticas não recomendáveis como automedicação com posologia e terapêutica inadequadas, podendo apresentar, como consequência, a intoxicação aguda por múltiplas substâncias, principal causa de hospitalizações encontrada neste estudo.

A maior ocorrência de óbitos no sexo masculino em controvérsia com o maior consumo de medicamentos por mulheres pode ser associada à circunstância de óbitos mais encontrada - autointoxicação intencional. Bochner \& Souza 36 afirmam que mulheres tentam mais contra a vida, enquanto os homens são mais bem-sucedidos nas tentativas de suicídio. Esse resultado está em consonância com o apresentado por Mota et al. ${ }^{14}$, que observaram maior prevalência de óbitos por intoxicações medicamentosas em homens $(53,9 \%)$ no seu estudo e com circunstância principalmente suicida $(57,2 \%)$.

O maior número de óbitos e hospitalizações e as tendências ascendentes encontradas nas regiões Sul, Sudeste e Centro-oeste podem ser explicados pela observação da renda per capita, da organização dos serviços de saúde e das distribuições de locais de aquisição de medicamentos nas diferentes regiões do Brasil. Estudos de base populacional sobre utilização de medicamentos no país apontam um maior consumo entre os indivíduos de maior poder aquisitivo 28, e o rendimento domiciliar per capita divulgado pelo IBGE em 2016 mostra os dez estados das regiões Sul, Sudeste e Centro-oeste, e mais o Distrito Federal, ocupando os 11 primeiros lugares na classificação decrescente de renda 37. Bertoldi et al. 28, em seu estudo sobre utilização de medicamentos no Brasil, concluiu que há uma grande variabilidade nas prevalências de consumo entre as regiões do país. Os resultados apontam que as regiões mais desfavorecidas apresentam as menores prevalências devido especialmente à maior dependência e ineficiência do sistema público para acesso aos medicamentos, além do provável menor número de locais para aquisição dos medicamentos no setor privado.

$\mathrm{Na}$ análise dos eventos por faixa etária, foram observadas maiores taxas de óbitos em indivíduos com mais de 59 anos. Diferentes estudos sobre consumo de medicamentos realizados no Brasil encontraram associação positiva entre o maior consumo e a idade elevada 22, o aumento da prevalência de doenças crônicas com a idade demanda maior utilização de medicamentos. Além disso, pessoas de idade avançada são mais sensíveis a efeitos adversos, interações medicamentosas e toxicidade. Assim, a prática da polifarmácia associada à maior suscetibilidade dos idosos a episódios de toxicidade favorece a ocorrência elevada de intoxicações e reações adversas a medicamentos nessa faixa etária.

As maiores taxas de hospitalização foram encontradas na faixa etária de 20 a 59 anos, tendo como principal causa a intoxicação aguda por múltiplas drogas ou substâncias psicoativas. Um dos motivos pode ser o fato de que esse grupo etário é passível de experimentar o aumento dos tratamentos medicamentosos contínuos de doenças crônicas, característicos da transição epidemiológica vivenciada no país. É interessante observar ainda que a maior causa de óbitos encontrada nessa faixa etária foi a autointoxicação intencional (24\%), o que pode sugerir um número significativo de tentativas de suicídio por intoxicações medicamentosas nessa população. Esse dado também foi encontrado por Mota et al. $14 \mathrm{em}$ seu estudo, o qual apontou maior taxa de óbitos por intoxicações medicamentosas na faixa etária de 20 a 59 anos, tendo como principal circunstância de intoxicação o suicídio.

Em separado da autointoxicação intencional, o uso irracional de medicamentos parece ter a considerável probabilidade de ser a causa do elevado número de hospitalizações encontrado nessa faixa etária. Esse fato pode ocorrer devido principalmente a fatores como a necessidade de aprimoramento da regulação da publicidade de medicamentos no país, a facilidade da aquisição sem prescrição médica, a escassez de iniciativas de desenvolvimento da atenção farmacêutica e, especialmente, o padrão de consumo de medicamentos pela população, caracterizado pela automedicação inapropriada, polifarmácia e uso indevido e indiscriminado de medicamentos 14 .

A análise de tendência por faixas etárias apresentou tendência de óbito e hospitalizações declinantes para menores de cinco anos. Estudos sobre intoxicações medicamentosas em crianças menores de cinco anos apontam vulnerabilidade dessa faixa etária relacionada a condições inerentes ao desenvolvimento infantil, como a curiosidade e exploração de ambientes utilizando todos os sentidos, na maioria das vezes levando à boca os objetos que encontram pelo caminho 38 , o que seria a causa do elevado número de intoxicações acidentais observado nessa faixa etária. O maior cuidado dos responsáveis com a criança quanto ao local de armazenamento de medicamentos no domicílio, de modo a evitar o acesso dela e, por consequência, evitar a intoxicação acidental que é característica dessa 
faixa etária, pode ser uma das causas do declínio de óbitos e hospitalizações observado em menores de cinco anos.

Devido à incerteza da eficácia e segurança dos medicamentos disponíveis para crianças e à carência de formulações apropriadas para essa faixa etária, a OMS lançou, em 2007, a campanha Make Medicines Child Size e publicou a primeira lista de medicamentos essenciais para crianças, objetivando a melhoria da disponibilidade e do acesso a medicamentos seguros e apropriados para crianças 39 . Desde então, medidas regulatórias e de incentivo ao desenvolvimento desses medicamentos têm ocorrido em diferentes países. A melhoria do desenvolvimento de medicamentos específicos para crianças também pode ser uma das causas da tendência declinante de óbitos e hospitalizações observada nessa faixa etária.

A tendência de óbitos apresentou-se ascendente para maiores de quatro anos. Essa observação pode ser decorrente do fato de que essa população não está mais sujeita às circunstâncias acidentais observadas para crianças menores de cinco anos e sim às circunstâncias de autointoxicação intencional e intoxicação por múltiplas drogas, como foi observado no estudo. Essas circunstâncias são geralmente de evolução mais grave do que as ocorrências de ingestão involuntária características dos primeiros anos de vida. Ainda segundo Bertoldi et al. 28, há um crescimento gradual da utilização de medicamentos à medida que aumentam a idade e os percentuais de tratamento de doenças crônicas. Isso pode tornar esses grupos etários mais suscetíveis à ocorrência de intoxicações e reações adversas a medicamentos.

A tendência de hospitalizações apresentou-se ascendente, para indivíduos de 20 a 59 anos, em consonância com os maiores coeficientes de hospitalizações encontrados para essa faixa etária no estudo. Aqui valem as mesmas observações relacionadas à característica de tratamentos medicamentosos contínuos nessa faixa, por advento do aumento de doenças crônicas vivenciado no país; e também o fato de que esse grupo é o mais acometido por casos de tentativa de suicídio por intoxicações medicamentosas, sendo essa a principal causa de óbitos observada nessa faixa etária.

As limitações encontradas foram relacionadas principalmente às fontes de dados. O não conhecimento das taxas de admissões hospitalares nos estabelecimentos de saúde privados, não abrangidos pelo SIH-SUS, pode levar à subestimação da verdadeira magnitude das taxas de hospitalização por intoxicações e reações adversas a medicamentos. O mau preenchimento das Declarações de Óbito (DO) e Autorizações de Internação Hospitalar (AIH), especialmente nos campos relacionados à causa de morte/internação, também pode subestimar os resultados encontrados. Uma das maiores limitações da utilização de dados secundários encontra-se no fato de que o pesquisador não tem controle sobre os possíveis erros de digitação ou registro. Além disso, há pouca quantidade de estudos prévios sobre o tema que tenham utilizado o SIM e o SIH-SUS, impedindo a comparação entre os resultados obtidos ao longo do tempo.

As intoxicações e reações adversas a medicamentos constituem uma importante causa de mortalidade e admissão hospitalar no Brasil, especialmente quando verificada a característica evitável da ocorrência desses eventos. A análise descritiva robusta das ocorrências no país é importante para caracterizar a distribuição desses eventos ao longo do tempo e promover o uso dos dados disponíveis nos sistemas de informação em saúde nacionais, promovendo concomitantemente a melhoria da qualidade desses dados e a conscientização da importância do monitoramento das ocorrências para o planejamento de medidas de controle e prevenção de novos casos. A descrição dos eventos também pretende cumprir a função de servir de subsídio para a iniciativa de realização de estudos analíticos sobre o tema. 


\section{Colaboradores}

G. A. S. Santos e A. C. Boing foram responsáveis pelo planejamento, organização, análise, discussão, redação e revisão final do artigo.

\section{Agradecimentos}

À Coordenação de Aperfeiçoamento de Pessoal de Nível Superior (Capes) pela bolsa concedida durante o curso de mestrado.

\section{Referências}

1. Cano FG, Rozenfeld S. Adverse drug events in hospitals: a systematic review. Cad Saúde Pública 2009; 25 Suppl 3:S360-72.

2. World Health Organization. The safety of medicines: a guide to detecting and reporting adverse drug reaction - why health professionals nedd to take action. Geneva: World Health Organization; 2008.

3. Agência Nacional de Vigilância Sanitária. Farmacovigilância: glossário. http://s.anvisa.gov. br/wps/s/r/ix (acessado em 07/Mar/2016).

4. Alves C, Batel-Marques F, Macedo AF. Data sources on drug safety evaluation: a review of recent published meta-analyses. Pharmacoepidemiol Drug Saf 2012; 21:21-33.

5. Lieber NSR, Ribeiro E. Reações adversas a medicamentos levando crianças a atendimento na emergência hospitalar. Rev Bras Epidemiol 2012; 15:265-74.

6. Otero MJ, Dominguez-Gil A. Acontecimentos adversos por medicamentos: uma patologia emergente. Farm Hosp 2000; 24:258-66.

7. Classen DC. Medical safety: moving from illusion to reality. JAMA 2003; 289:1154-6.

8. Winterstein AG, Sauer BD, Hepler CD, Poole C. Preventable drug related hospital admission. Ann Pharmacother 2002; 36:1238-48.

9. Richardson R, Charters T, King N, Harper S. Trends in educational inequalities in drug poisoning mortality: United States, 1994-2010. Am J Public Health 2015; 105:1859-65.

10. Mowry JB, Spyker DA, Brooks DE, McMillan N, Schauben JL. 2014 Annual Report of the American Association of Poison Control Centers' National Poison Data System (NPDS): 32nd Annual Report. Clin Toxicol (Phila) 2015; 53:962-1147.

11. Corrêa AD, Caminha JR, Souza CAM, Alves LA. Uma abordagem sobre o uso de medicamentos nos livros didáticos de biologia como estratégia de promoção de saúde. Ciênc Saúde Coletiva 2013; 18:3071-81.

12. Sistema Nacional de Informações Toxicológicas (SINITOX). Registro de intoxicações no Brasil, 2012. http://sinitox.icict.fiocruz.br/ sites/sinitox.icict.fiocruz.br/files//Tabela\%20 3_2012.pdf (acessado em 04/Jan/2016).

13. Paula TC, Bochner R, Montilla DER. Análise clínica e epidemiológica das internações hospitalares de idosos decorrentes de intoxicações e efeitos adversos de medicamentos, Brasil, de 2004 a 2008. Rev Bras Epidemiol 2012; 15:828-44.

14. Mota DM, Melo JRR, Freitas DRC, Machado M. Perfil da mortalidade por intoxicação com medicamentos no Brasil, 1996-2005: retrato de uma década. Ciênc Saúde Coletiva 2012; 17:61-70.

15. Lessa MA, Bochner R. Análise das internações hospitalares de crianças menores de um ano relacionadas a intoxicações e efeitos adversos de medicamentos no Brasil. Rev Bras Epidemiol 2008; 11:660-74. 
16. Ahmad OB, Boschi-Pinto C, Lopez AD, Murray CJL, Lozano $\mathrm{R}$, Inoue $\mathrm{M}$. Age standardization of rates: a new WHO standard. Geneva: World Health Organaization; 2001.

17. Gaynor PE, Kirkpatrick RC. Introduction to time-series modeling and forecasting in business and economics. New York: McGraw-Hill; 1994.

18. Beijer HJ, Blaey CJ. Hospitalizations caused by adverse drug reactions (ADR): a meta-analysis of observational studies. Pharm World Sci 2002; 24:46-54.

19. Bouvy JC, Bruin MC, Koopmanschap MA. Epidemiology of adverse drug reactions in Europe: a review of recent observational studies. Drug Saf 2015; 38:437-53.

20. Silva AEBC, Reis AMM, Miasso AI, Santos JO, Cassiani SHB. Adverse drug events in a sentinel hospital in the state of Goiás, Brazil Rev Latinoam Enferm 2011; 19:378-86.

21. Loução AS, Sanches ACC, Carraro CB. Perfil das reações adversas a medicamentos notificadas em um hospital universitário. Revista Brasileira de Farmácia Hospitalar e Serviços de Saúde 2015; 6:12-7.

22. Ramos LR, Tavares NUL, Bertoldi AD, Farias MR, Oliveira MA, Luiza VL, et al. Polifarmácia e polimorbidade em idosos no Brasil: um desafio em saúde pública. Rev Saúde Pública 2016; 50 Suppl 2:9s.

23. Loyola Filho AI, Uchoa E, Guerra HL, Firmo JOA, Lima-Costa MF. Prevalência e fatores associados à automedicação: resultados do projeto Bambuí. Rev Saúde Pública 2002; 36:5562.

24. Bertoldi AD, Barros AJD, Hallal PC, Lima RC. Utilização de medicamentos em adultos: prevalência e determinantes individuais. Rev Saúde Pública 2004; 38:228-38.

25. Arrais PSD, Brito LL, Barreto ML, Coelho HLL. Prevalência e fatores determinantes do consumo de medicamentos no Município de Fortaleza, Ceará, Brasil. Cad Saúde Pública 2005; 21:1737-46.

26. Ribeiro AQ, Rozenfeld S, Klein CH, César CC, Acurcio FA. Inquérito sobre o uso de medicamentos por idosos aposentados, Belo Horizonte, MG. Rev Saúde Pública 2008; 42:724-32.

27. Silveira EA, Dalastra L, Pagotto V. Polifarmácia, doenças crônicas e marcadores nutricionais em idosos. Rev Bras Epidemiol 2014; 17:818-29.

28. Bertoldi AD, Pizzol TS, Ramos LR, Mengue SS, Luiza VL, Tavares NUL, et al. Perfil sociodemográfico dos usuários de medicamentos no Brasil: resultados da PNAUM 2014. Rev Saúde Pública 2016; 50 Suppl 2:5s.
29. Juurlink DN, Mandani M, Kopp A, Laupacis A, Redelmeier DA. Drug-drug interactins among elderly patients hospitalized for drug toxicity. JAMA 2006; 289:1652-8.

30. van der Hooft MH, Sturkenboom MC, van Grootheest K, Kingma HJ, Stricker BHCH. Adverse drug reaction-related hospitalizations: a nationwide study in The Netherlands. Drug Saf 2006; 29:161-8.

31. Queneau P, Bannwath B, Carpentier F, Guliana JM, Bouget J, Trombert B, et al. Emergence department visits caused by adverse drug events. Drug Saf 2007; 30:81-8.

32. Costa KS, Barros MBA, Francisco PMSB, César CLG, Goldbaum M, Carandina L. Utilização de medicamentos e fatores associados: um estudo de base populacional no Município de Campinas, São Paulo, Brasil. Cad Saúde Pública 2011; 27:649-58.

33. Francisco PMSB, Bastos TF, Costa KS, Prado MAMB, Barros MBA. The use of medication and associated factors among adults living in Campinas, São Paulo, Brazil: differences between men and women. Ciênc Saúde Coletiva 2014; 19:4909-21.

34. Galvão TF, Silva MT, Gross R, Pereira MG. Medication use in adults living in Brasilia, Brazil: a cross-sectional, population-based study. Pharmacoepidemiol Drug Saf 2014; 23:507-14.

35. Tavares NUL, Costa KS, Mengue SS, Vieira MLFP, Malta DC, Silva JB. Uso de medicamentos para tratamento de doenças crônicas não transmissíveis no Brasil: resultados da Pesquisa Nacional de Saúde, 2013. Epidemiol Serv Saúde 2015; 24:315-23.

36. Bochner R, Souza VMFA. Panorama das intoxicações e envenenamentos registrados no Brasil pelo Sistema Nacional de Informações Tóxico-Farmacológicas (SINITOX). Revista Racine 2008; 106:44-58.

37. Instituto Brasileiro de Geografia e Estatística. IBGE divulga o rendimento domiciliar per capita 2016. ftp://ftp.ibge.gov.br/Trabalho_e_Ren dimento/Pesquisa_Nacional_por_Amostra_ de_Domicilios_continua/Renda_domiciliar_ per_capita/Renda_domiciliar_per_capita_ 2016.pdf (acessado em 23/Abr/2017).

38. Domingos SM, Borghesan NBA, Merino MFGL, Higarashi IH. Internações por intoxicação de crianças de zero a 14 anos em hospital de ensino no Sul do Brasil, 2006-2011. Epidemiol Serv Saúde 2016; 25:343-50.

39. World Health Organization. Promoting safety of medicines for children. Geneva: World Health Organization; 2007. 


\section{Abstract}

In recent years, drug poisoning and adverse reactions have been an important cause of hospitalization and mortality and a major public health issue. The aim of this study was to describe trends in hospitalizations and deaths from drug poisoning and adverse reactions from 2000 to 2014. Data were from the Mortality Information System (SIM) and Information System on Authorizations for Hospital Admissions of the Brazilian Unified National Health System (SIH-SUS), and the study population was analyzed by sex, region of residence, and age bracket. Events were defined according to ICD-10 codes. The analysis of trends in the historical series used Prais-Winsten generalized linear regression with correction for first order autocorrelation effect. Both the hospitalizations and deaths during the study period showed upward trends. The analysis by regions maintained the upward trends for both events in the South and Southeast regions. Analysis according to age brackets showed downward trends in children under five years in both events, upward trend in deaths in individuals over four years of age, and an upward trend in hospitalizations in the age bracket from 20 to 59 years. Drug poisoning and adverse reactions contribute significantly to hospitalizations and deaths in Brazil, and the SIM and SIH-SUS are data sources with satisfactory quality for population-based studies on hospital morbidity and mortality in the country.

Intoxication; Drug-Related Side Effects and Adverse Reactions; Mortality Registries;

Hospital Information Systems

\section{Resumen}

Durante los últimos años, las intoxicaciones y reacciones adversas a medicamentos se han convertido en una importante causa de hospitalización $y$ mortalidad, constituyendo un tema de gran relevancia para la salud pública. El objetivo de este estudio fue describir la tendencia de mortalidad $y$ hospitalizaciones por estos riesgos sanitarios en Brasil, entre los años 2000 y 2014. Los datos utilizados provinieron de los sistemas SIM (Sistema de Infomaciones sobre Mortalidad) y SIH-SUS (Sistema de Informaciones Hospilatarias del Sistema Único de Salud), y la población se analizó según sexo, región de residencia y franja de edad. Para la definición de los eventos, se utilizaron códigos CID-10. El análisis de tendencia de la serie histórica se realizó por regresión lineal generalizada, mediante el método de Prais-Winsten, con corrección del efecto de autocorrelación de primer orden. Tanto los óbitos como las hospitalizaciones ocurridas durante el período estudiado presentaron una tendencia ascendente. En el análisis por regiones, las tendencias se mantuvieron ascendentes para ambos eventos en las regiones Sudeste y Sur. El análisis por franja de edad mostró tendencias declinantes para menores de cinco años en los dos eventos, tendencia de óbitos ascendente para mayores de cuatro años y tendencia de hospitalizaciones ascendente para la franja de 20 a 59 años. Las intoxicaciones y reacciones adversas a medicamentos presentan una considerable contribución a la ocurrencia de óbitos y hospitalizaciones en Brasil, y los sistemas de información SIM y SIH-SUS son fuentes de datos de calidad satisfactoria para estudios de base poblacional sobre mortalidad y morbilidad hospitalaria en el país

Intoxicación; Efectos Colaterales y Reacciones Adversas Relacionados con Medicamentos; Registros de Mortalidad; Sistemas de Información en Hospital
Recebido em 13/Jun/2017

Versão final reapresentada em 05/Set/2017 Aprovado em 18/Dez/2017 